\title{
Effects of Escitalopram on Anxiety in Patients with Acute Coronary Syndrome: A Randomized Controlled Trial
}

\author{
Hee-Ju Kang ${ }^{1}$, Kyung-Yeol Bae ${ }^{1}$, Sung-Wan Kim ${ }^{1}$, II-Seon Shin ${ }^{1}$, Young Joon Hong ${ }^{2}$, Youngkeun Ahn ${ }^{2}$, \\ Myung Ho Jeong ${ }^{2}$, Jin-Sang Yoon ${ }^{1}$, Jae-Min Kim ${ }^{1}$ \\ Departments of ${ }^{1}$ Psychiatry and ${ }^{2}$ Cardiology, Chonnam National University Medical School, Gwangju, Korea
}

\begin{abstract}
Objective: There are no evidence-based practices for treating anxiety in patients with acute coronary syndrome (ACS). Thus, we investigated the effects of escitalopram on anxiety in this population.

Methods: We enrolled 217 patients with ACS who met Diagnostic and Statistical Manual of Mental Disorders, 4th edition (DSM-IV) criteria for depressive disorders into a randomized double-blind placebo-controlled study. Patients received either escitalopram or placebo over a 24-week period. Anxiety symptoms were evaluated using the Hospital Anxiety and Depression Scale-anxiety subscale (HADS-A). A HADS-A score $>7$ was classified as an anxiety disorder. Baseline evaluations included sociodemographic and clinical characteristics, such as depressive symptoms, cardiovascular risk factors, and current cardiac status.

Results: Independent of improvements in depression and without any differences in safety profiles, escitalopram treatment was significantly more efficacious in treating and reducing anxiety than placebo over a 24-week period.

Conclusion: Escitalopram can be recommended as an effective and safe treatment option for anxiety in patients with ACS.
\end{abstract}

KEY WORDS: Acute coronary syndrome; Anxiety; Depression; Escitalopram; Double-blind study.

\section{INTRODUCTION}

In recent years, a number of studies have demonstrated an association between depression and a poor prognosis in patients with acute coronary syndrome (ACS). ${ }^{1,2)}$ Anxiety is a well-established prevalent comorbid condition accompanying depression in the general population and has been associated with a poor prognosis in patients with depression. ${ }^{3,4)}$ As a result, the American Heart Association has recommended that patients who positively screen for depression also be assessed for anxiety. ${ }^{5}$ Additionally, previous study of patients in ACS found that anxiety was highly comorbid with depression and their independent contribution to poor cardiac prognosis. ${ }^{6}$ However, studies on the treatment effects of anxiety are rare in patients with ACS. This is in contrast to extensive studies investigating outcomes of patients with ACS and depression. ${ }^{\text {7) }}$

Recently, our research group conducted a 24 -week,

\footnotetext{
Received: July 25, 2016 / Revised: August 12, 2016

Accepted: August 13, 2016

Address for correspondence: Jae-Min Kim, MD, PhD

Department of Psychiatry, Chonnam National University Medical

School, 160 Baekseo-ro, Dong-gu, Gwangju 61469, Korea

Tel: +82-62-220-6143, Fax: +82-62-225-2351

E-mail:.jmkim@chonnam.ac.kr
}

double-blind, placebo-controlled trial assessing the efficacy and safety of escitalopram for treating depressive disorders in ACS patients: the Escitalopram for DEPression in ACS (EsDEPACS) study (ClinicalTrial.gov registry number: NCT00419471). This study found that escitalopram was superior to placebo for treating depression without any differences in safety profiles. ${ }^{8)}$ Escitalopram is known to be effective for treating anxiety in general populations, ${ }^{9)}$ but has yet to be formally assessed in ACS patients. Thus, the present study investigated the efficacy and safety of escitalopram for treating anxiety in ACS patients with depressive disorders. To accomplish this, a secondary analysis of data from the EsDEPACS study was performed.

\section{METHODS}

\section{Study Overview and Escitalopram Intervention}

We used data from the aforementioned EsDEPACS study in our analyses. Note that the detailed study design and findings of the EsDEPACS trial were published previously. ${ }^{8}$ In brief, 1,152 consecutive ACS patients were recruited from the Department of Cardiology at Chonnam National University Hospital in Gwangju,

(a) This is an Open-Access article distributed under the terms of the Creative Commons Attribution Non-Commercial License (http://creativecommons.org/licenses/by-nc/4.0) which permits unrestricted non-commercial use, distribution, and reproduction in any medium, provided the original work is properly cited. 
South Korea. Of these, 446 were diagnosed with minor or major depressive disorders using the criteria of the Diagnostic and Statistical Manual of Mental Disorders, 4th edition (DSM-IV), ${ }^{10)}$ between 1 week and 3 months after presenting with ACS. Of these patients, 300 agreed to participate in the EsDEPACS trial and were randomly assigned to either the escitalopram $(n=149)$ or the placebo $(n=151)$ group. The participant sample selected for analysis included a total of 217 patients who were assessed at least once after baseline (108 escitalopram, 109 placebo). Flexible doses of escitalopram $(5,10,15$, or $20 \mathrm{mg})$ or matched placebo were assigned based on patient response and tolerability by the clinical decision of the investigators. Assessments were performed at baseline and at weeks 4, 8, 12, 16, 20, and 24. This EsDEPACS study was registered in ClinicalTrials.gov (NCT00419471). Informed written consent was obtained from all participants, and the study was approved by the Chonnam National University Hospital Institutional Review Board (06-026).

\section{Anxiety Symptoms and Baseline Evaluation}

In this analysis, the Hospital Anxiety and Depression Scale (HADS) was used to identify symptoms of anxiety. The HADS, which was previously developed to estimate depression and anxiety disorders among physically ill patients, ${ }^{11)}$ consists of an anxiety subscale (HADS-A) and a depression subscale (HADS-D). Anxiety symptoms in ACS patients were evaluated using the HADS-A, and a HADS-A score $>7$ was defined as reflective of an anxiety disorder. $^{12)}$

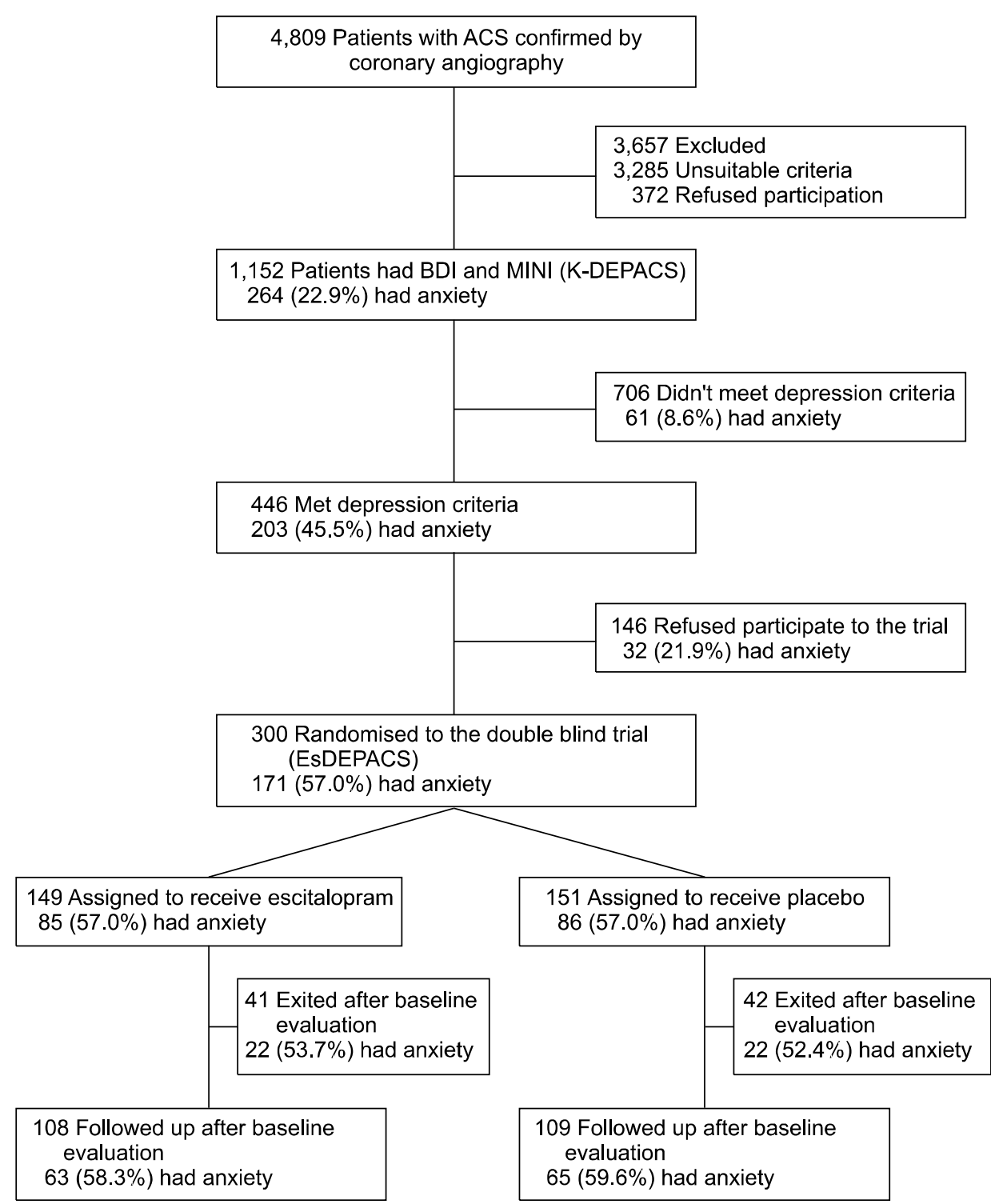

Fig. 1. Participant recruitment process and prevalence of anxiety. ACS, acute coronary syndrome: $\mathrm{BDI}$, Beck Depression Inventory; MINI, Mini-International Neuropsychiatric Interview; K-DEPACS, Korean DEPression in Acute Coronary Syndrome study; EsDEPACS, Escitalopram for DEPression in Acute Coronary Syndrome study. 
The following were comprehensively evaluated at baseline: sociodemographic factors, depressive characteristics, cardiovascular risk factors, and current cardiac status: 1) The sociodemographic factors included age, gender, education level, living status (living alone or not), accommodation tenure (owned or rented), and current occupation (employed or not). 2) The depression characteristics included previous and family histories of depression and scores on the Montgomery Asberg Depression Rating Scale (MADRS). ${ }^{13)}$ 3) Cardiovascular risk factors included previous and family histories of ACS, diagnosed hypertension, diabetes mellitus, presence of hypercholesterolemia (fasting serum total cholesterol level $>200 \mathrm{mg} / \mathrm{dl}$ ), obesity according to body mass index, and reported current smoking status. 4) Current cardiac status included the Killip classification, ${ }^{14)}$ left ventricular ejection fraction, and serum levels of troponin I and creatine kinase-MB (CK-MB).

\section{Statistical Analysis}

The baseline characteristics of patients treated with escitalopram or placebo were compared with Student's $t$ tests, chi-square $\left(\chi^{2}\right)$ tests, or Fisher's exact tests, where appropriate. In patients with an anxiety disorder, remission was defined as a HADS-A score $\leq 7$ and assessed at each follow-up visit. Cox proportional hazards models were applied to determine the hazard ratio (HR; 95\% confidence interval $[\mathrm{CI}]$ ) to assess the timing to remission, adjusting for baseline HADS-A scores and changes in MADRS. For clinical relevance, the number-neededto-treat (NNT) was calculated. Additionally, a repeated measures analysis of covariance with the same adjusted model was conducted on data from all subjects undergoing the drug trial to estimate group-by-time interactions for the HADS-A scores. To account for missing data, a multiple imputation by chained equations was applied after adjusting for relevant covariates. All statistical analyses were performed using IBM SPSS Statistics ver. 21.0 (IBM Co., Armonk, NY, USA) and STATA 12.0 software (Stata Co., College Station, TX, USA).

\section{RESULTS}

\section{Recruitment}

The recruitment process and prevalence rates of anxiety disorder at each assessment included in this study are described in Figure 1. Of the 446 ACS patients diagnosed with depressive disorder, 300 participated in the EsDEPACS study and were randomized to either the esci- talopram or placebo group. Of those enrolled, 83 (27.7\%) exited the study due to lack of follow up after baseline evaluation. Thus, the remaining 217 (108 on escitalopram and 109 on placebo) patients were used for analysis. Baseline characteristics of both escitalopram and placebo groups are summarized in Supplementary Table 1. No significant differences between the two groups were found in any characteristic (all $p>0.2$ ). Compared with patients who completed follow-up assessments after baseline evaluation, patients who exited the study had significantly higher CK-MB levels ( $p=0.043$ ). There were no other significant differences in the characteristics of these two groups. The detailed comparisons are also described in Supplementary Table 1.

\section{Treatment Effect of Escitalopram on Anxiety Disorder}

An anxiety disorder was present in $63(58.3 \%)$ and 65 $(59.6 \%)$ patients in the escitalopram and placebo groups, respectively. There was no significant difference in baseline characteristics between the two groups (all $p$ values $>0.05)$. Remission in the escitalopram group occurred significantly earlier versus placebo after adjustment for baseline HADS-A scores (HR, 1.89; 95\% CI, 1.20-2.99) with $\mathrm{NNT}=5$ at week 20 and 4 at week 24 . After including

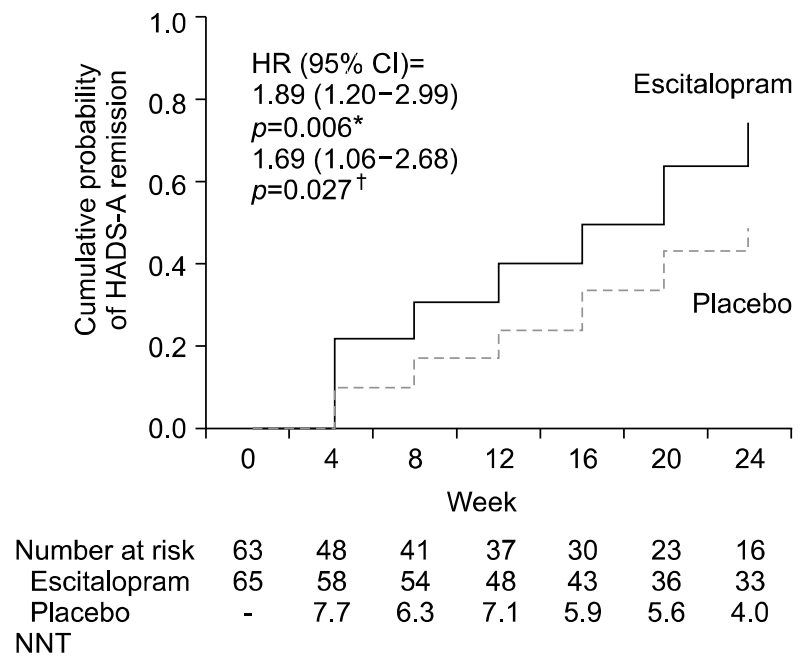

Fig. 2. Time to Hospital Anxiety Depression Scale-anxiety subscale (HADS-A) remissions and numbers needed-to-treat (NNT) between the treatment groups $(n=128)$. Cumulative proportions of participants that achieved remission (HADS-A score $\leq 7$ ) were driven from Kaplan Meyer models. The hazard ratio (HR), 95\% confidence interval $(\mathrm{Cl})$, and $p$ values were drawn from Cox regression HR tests after adjusting for baseline HADS-A scores* and further adjusted for changes in Montgomery Asberg Depression Rating Scale scores ${ }^{\dagger}$. NNT indicates the number of patients in the escitalopram group that would be required for one more person to achieve remission. 


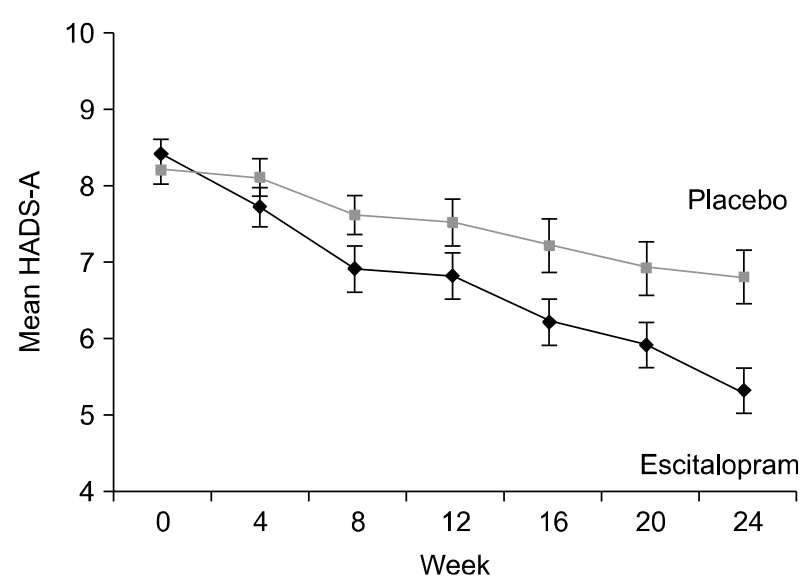

Fig. 3. Adjusted mean scores on the Hospital Anxiety Depression Scale-anxiety subscale (HADS-A) in the 24 week double blind treatment of escitalopram and placebo $(n=217)$.

Statistical coefficients were driven from repeated measures analysis of covariance to calculate group by time interactions on the HADS-A. Statistics adjusted for baseline HADS-A scores: $F=12.366, p=0.001$; and further adjusted for changes in Montgomery Asberg Depression Rating Scale scores: $F=4.467, p=0.036$.

changes in MADRS scores from baseline to week 24, the strength of association was weakened but remained significant (HR, 1.69; 95\% CI, 1.06-2.68) (Fig. 2). According to our analysis including all randomized subjects $(n=217)$, the escitalopram (mean \pm standard deviation [SD], 8.4 \pm 2.6$)$ and placebo (8.1 \pm 2.2$)$ groups did not differ with regard to baseline HADS-A scores. However, the groups did significantly differ in changes in HADS-A scores (mean $\pm \mathrm{SD}$ : escitalopram group, $-3.0 \pm 2.1$; placebo group, $-1.3 \pm 1.8$ ) in that escitalopram significantly reduced anxiety symptoms compared with placebo. Group-by-time interactions for HADS-A scores were significant after adjusting for baseline HADS-A scores $(\mathrm{F}=12.366, p=0.001)$ and further adjusting for changes in MADRS scores $(\mathrm{F}=4.467, p=0.036$; Fig. 3 ). There were no significant differences in drug treatment between the two groups regarding dose, duration, concomitant medications, discontinuation, or adverse events (Supplementary Table 2).

\section{DISCUSSION}

The findings of this 24-week randomized placebo-controlled trial suggested that escitalopram treatment led to significantly earlier remission of anxiety than did placebo in patients with ACS. Furthermore, it was demonstrated that escitalopram significantly reduced anxiety symptoms compared with placebo. It should be noted that the im- provement in anxiety symptoms by escitalopram was independent of improvements in depression. Moreover, there were no differences in the safety profiles of the escitalopram and placebo groups.

Our analyses demonstrated that 24 weeks of escitalopram intervention was significantly more effective for remitting anxiety disorder and reducing anxiety symptoms. This effect was independent of improvements in depression in patients with recent ACS and was not associated with prominent adverse events. Similar to findings in the general population, ${ }^{9)}$ escitalopram is also effective for managing anxiety in patients with ACS. Because of the notable burden of anxiety on patients with ACS, it is crucial to ensure effective therapeutic strategies are developed.

It should be noted that this trial was originally designed to treat depression as a primary outcome. As such, all participants were enrolled after diagnosis with depressive disorders. However, anxiety is frequently comorbid with depression in both the general and ACS populations. ${ }^{7,9)}$ Our findings support the effects of escitalopram on patients with anxiety and depressive disorders in the general population. ${ }^{9,15)}$ Additionally, more caution is needed to interpret our findings due to follow-up attrition. There were 83 patients (of a total of 300 randomized participants) who exited the study and were lost to follow up. These patients had significantly higher serum CK-MB levels compared with those who completed follow-up assessments. It can be assumed that ACS of greater severity might have influenced attrition and thereby affected our findings. Finally, the potential risk of developing QTc prolongation should be considered for treating anxiety with escitalopram in patients with ACS. Recently, studies have suggested that higher dose of citalopram and escitalopram is associated with QTc prolongation. ${ }^{16,17)}$ Although our study found no difference in QTc duration between escitalopram and placebo, relatively low dose of escitalopram in our study might contribute to this findings despite similar findings were suggested in the antidepressant trial of depression in ACS with higher citalopram doses. ${ }^{18)}$

Despite these limitations, this is the first randomized placebo-controlled trial to investigate the efficacy and safety of antidepressants, specifically escitalopram, for treating anxiety improvement in patients with ACS. Comprehensive evaluations were conducted, and well-validated psychiatric assessments were used in this study. Despite its high prevalence and negative impact on patients, there are currently no evidence-based practices for treating anxiety in the ACS population. The present findings indicate that escitalopram can be recommended as an 
effective and safe treatment option for treating anxiety in ACS patients, but larger studies from multicenter settings would increase their generalizability.

\section{Acknowledgments}

The study was supported by a grant of the Korea Health Technology R\&D Project through the Korea Health Industry Development Institute (KHIDI), funded by Ministry of Health \& Welfare, Republic of Korea (HC15C1405).

\section{REFERENCES}

1. Lichtman JH, Froelicher ES, Blumenthal JA, Carney RM, Doering LV, Frasure-Smith N, et al. Depression as a risk factor for poor prognosis among patients with acute coronary syndrome: systematic review and recommendations: a scientific statement from the American Heart Association. Circulation 2014;129:1350-1369.

2. Meijer A, Conradi HJ, Bos EH, Thombs BD, van Melle JP, de Jonge P. Prognostic association of depression following myocardial infarction with mortality and cardiovascular events: a meta-analysis of 25 years of research. Gen Hosp Psychiatry 2011;33:203-216.

3. Kessler RC, Chiu WT, Demler O, Merikangas KR, Walters EE. Prevalence, severity, and comorbidity of 12-month DSM-IV disorders in the National Comorbidity Survey Replication. Arch Gen Psychiatry 2005;62:617-627.

4. Fava M, Rush AJ, Alpert JE, Balasubramani GK, Wisniewski SR, Carmin CN, et al. Difference in treatment outcome in outpatients with anxious versus nonanxious depression: a STAR ${ }^{*} D$ report. Am J Psychiatry 2008;165: 342-351.

5. Lichtman JH, Bigger JT Jr, Blumenthal JA, Frasure-Smith $\mathrm{N}$, Kaufmann PG, Lespérance F, et al. Depression and coronary heart disease: recommendations for screening, referral, and treatment: a science advisory from the American Heart Association Prevention Committee of the Council on cardiovascular nursing, council on clinical cardiology, council on epidemiology and prevention, and interdisciplinary council on quality of care and outcomes research: endorsed by the American Psychiatric Association. Circulation 2008;118:1768-1775.

6. Cepeda-Valery B, Cheong AP, Lee A, Yan BP. Measuring health related quality of life in coronary heart disease: the importance of feeling well. Int J Cardiol 2011;149:4-9.

7. Tully PJ, Cosh SM, Baumeister H. The anxious heart in whose mind? A systematic review and meta-regression of factors associated with anxiety disorder diagnosis, treatment and morbidity risk in coronary heart disease. $J$ Psychosom Res 2014;77:439-448.

8. Kim JM, Bae KY, Stewart R, Jung BO, Kang HJ, Kim SW, et al. Escitalopram treatment for depressive disorder following acute coronary syndrome: a 24-week double-blind, placebocontrolled trial. J Clin Psychiatry 2015;76:62-68.

9. Thaler KJ, Morgan LC, Van Noord M, Gaynes BN, Hansen RA, Lux LJ, et al. Comparative effectiveness of secondgeneration antidepressants for accompanying anxiety, insomnia, and pain in depressed patients: a systematic review. Depress Anxiety 2012;29:495-505.

10. American Psychiatric Association. Diagnostic and Statistical Manual of Mental Disorders, fourth edition (DSM-IV). Washington, DC:American Psychiatric Press Inc;1994.

11. Zigmond AS, Snaith RP. The hospital anxiety and depression scale. Acta Psychiatr Scand 1983;67:361-370.

12. Bjelland I, Dahl AA, Haug TT, Neckelmann D. The validity of the Hospital Anxiety and Depression Scale. An updated literature review. J Psychosom Res 2002;52:69-77.

13. Montgomery SA, Asberg M. A new depression scale designed to be sensitive to change. Br J Psychiatry 1979;134:382389.

14. Killip T 3rd, Kimball JT. Treatment of myocardial infarction in a coronary care unit. A two year experience with 250 patients. Am J Cardiol 1967;20:457-464.

15. Choi KW, Woo JM, Kim YR, Lee SH, Lee SY, Kim EJ, et al. Long-term escitalopram treatment in Korean patients with panic disorder: a prospective, naturalistic, open-label, multicenter trial. Clin Psychopharmacol Neurosci 2012;10: 44-48.

16. Pae CU, Wang SM, Lee SJ, Han C, Patkar AA, Masand PS. Antidepressant and $Q T$ interval prolongation, how should we look at this issue? Focus on citalopram. Expert Opin Drug Saf 2014;13:197-205.

17. Wang SM, Pae CU. How much to worry about the FDA warning in the use of citalopram? Expert Rev Neurother 2013;13:883-886.

18. Lespérance F, Frasure-Smith N, Koszycki D, Laliberté MA, van Zyl LT, Baker B, et al. Effects of citalopram and interpersonal psychotherapy on depression in patients with coronary artery disease: the Canadian Cardiac Randomized Evaluation of Antidepressant and Psychotherapy Efficacy (CREATE) trial. JAMA 2007;297:367-379. 